\title{
Macrophage Autophagy and Oxidative Stress: An Ultrastructural and Immunoelectron Microscopical Study
}

\author{
Ida Perrotta, ${ }^{1}$ Valentina Carito, ${ }^{2}$ Emilio Russo, ${ }^{3}$ Sandro Tripepi, ${ }^{1}$ \\ Saveria Aquila, ${ }^{2}$ and Giuseppe Donato ${ }^{4}$ \\ ${ }^{1}$ Department of Ecology, Faculty of Mathematical, Physical and Natural Sciences (SMFN), University of Calabria, \\ 87036 Cosenza, Italy \\ ${ }^{2}$ Department of Pharmaco-Biology, Faculty of Pharmacy, University of Calabria, 87036 Cosenza, Italy \\ ${ }^{3}$ Department of Experimental and Clinical Medicine, School of Medicine, University Magna Graecia, 88100 Catanzaro, Italy \\ ${ }^{4}$ Department of Pathology, School of Medicine, University Magna Graecia, 88100 Catanzaro, Italy
}

Correspondence should be addressed to Ida Perrotta, idaperrotta@yahoo.it

Received 26 May 2011; Revised 27 June 2011; Accepted 27 June 2011

Academic Editor: Pranela Rameshwar

Copyright (C) 2011 Ida Perrotta et al. This is an open access article distributed under the Creative Commons Attribution License, which permits unrestricted use, distribution, and reproduction in any medium, provided the original work is properly cited.

\begin{abstract}
The word autophagy broadly refers to the cellular catabolic processes that lead to the removal of damaged cytosolic proteins or cell organelles through lysosomes. Although autophagy is often observed during programmed cell death, it may also serve as a cell survival mechanism. Accumulation of reactive oxygen species within tissues and cells induces various defense mechanisms or programmed cell death. It has been shown that, besides inducing apoptosis, oxidative stress can also induce autophagy. To date, however, the regulation of autophagy in response to oxidative stress remains largely elusive and poorly understood. Therefore, the present study was designed to examine the ratio between oxidative stress and autophagy in macrophages after oxidant exposure (AAPH) and to investigate the ultrastructural localization of beclin-1, a protein essential for autophagy, under basal and stressful conditions. Our data provide evidence that oxidative stress induces autophagy in macrophages. We demonstrate, for the first time by immunoelectron microscopy, the subcellular localization of beclin-1 in autophagic cells.
\end{abstract}

\section{Introduction}

The term autophagy (from the Greek "auto" for "self" and "phagein" meaning "to eat"), was first used in the 1960s by Christian de Duve and refers to a series of intracellular processes that lead to the removal of cytosolic proteins or entire cell organelles through lysosomes [1]. In mammalian cells, the three main autophagic pathways, macroautophagy, microautophagy, and chaperone-mediated autophagy (CMA) are frequently interconnected and share some common components. Macroautophagy (hereafter simply referred to as autophagy) has been originally described as a cellular adaptation to starvation involving the sequestration of cytoplasmic proteins and organelles into a closed, double membrane structure, called the autophagosome that consequently fuses with lysosomes to form an autolysosome, where the final hydrolytic degradation of the engulfed cytoplasmic material takes place [2-4]. Although autophagy appears to operate broadly as a cell survival mechanism, a dysregulation of autophagic flux may also be associated with cellular toxicity and may potentially contribute to the development of pathological conditions through excessive depletion of essential cytoplasmic components [5]. The regulation of autophagy has been extensively studied in the past few years especially under nutrient deprivation conditions; however, most mammalian autophagy proteins are still not characterized at all and very little is known about the autophagic response to stress and/or pathological damage [6-8]. Beclin-1, a mammalian orthologue of the yeast Apg6/Vps30 gene, is the first identified mammalian gene with a role in mediating autophagy and appears to act as a nexus point between autophagy, endosomal, and perhaps also cell death pathways $[9,10]$. Although the molecular properties of beclin-1 have been characterized, its subcellular localization has not been unequivocally determined $[11,12]$. Reactive oxygen species (ROS) have a complex relationship with autophagy and 
beclin-1 expression [13-15]. ROS (superoxide, hydrogen peroxide, hydroxyl radical, and other related compounds) are produced continuously in cells as consequence of both enzymatic and nonenzymatic reactions and regulate a diverse array of physiological functions [16]. However, when under certain conditions the production of ROS becomes excessive, the resultant oxidative stress can negatively affect biomolecules (such as proteins or DNA) and can alter the functional and structural integrity of biological tissues [17]. ROS not only cause a direct damage of cell constituents, but also may serve as important stimuli of autophagy becoming involved in several pathways that regulate both cell survival and death [18]. Even if different methodological approaches have been adopted to detect autophagy, electron microscopy remains currently the most accurate technique that allows the visualization of the morphological events and molecular mechanisms of autophagy and provides a faithful demonstration of the ongoing of autophagic activity within cells. In the present study we show, for the first time by immunoelectron microscopy, the subcellular localization of beclin-1 in autophagic cells and further evidence that autophagy is a cellular mechanism closely related to oxidative stress.

\section{Materials and Methods}

2.1. Macrophage Isolation and Culture Conditions. Peripheral blood mononuclear cells (PBMCs) were obtained from male healthy donors (25-30 years old). Buffy coat preparations from healthy donors were diluted $1: 1$ with phosphatebuffered saline solution (PBS) and centrifuged over FicollPaque solution (GE Health Care, Milan, Italy). Monocytes were separated from lymphocytes by adherence as described previously with minor modifications [19]. PBMCs were cultured at $37^{\circ} \mathrm{C}$ in humidified atmosphere enriched with $5 \% \mathrm{CO}_{2}$ in RPMI 1640 supplemented with $1 \%(\mathrm{v} / \mathrm{v})$ of penicillin/streptomycin and $10 \%(\mathrm{v} / \mathrm{v})$ of fetal bovine serum heat-inactivated and endotoxin free. After 7 days of culture, nonadherent cells were removed by repeated washings with warm medium. Macrophages obtained with this method resulted in $>95 \%$ of purity by cytofluorimetric analysis. To monitor the ability of oxidative stress to induce autophagy, macrophages were incubated with free radical generator 2,2-azobis (2-amidinopro-pane) dihydrochloride (AAPH, $5 \mathrm{mmol} / \mathrm{L}$ ) in Dulbecco's modified Eagle's medium without serum at $37^{\circ} \mathrm{C}$ in the dark for $5 \mathrm{~h}$.

After this incubation period, cells (untreated controls and AAPH-treated macrophages) were detached with trypsin treatment, concentrated by gentle centrifugation, and adequately processed for electron microscopy.

2.2. Transmission Electron Microscopy. For routine transmission electron microscopy, cell pellets were fixed in 3\% glutaraldehyde solution prepared in $0.1 \mathrm{M}$ phosphate buffer at $4^{\circ} \mathrm{C}$, postfixed in osmium tetroxide $(3 \%)$ for 2 hours, dehydrated in graded acetone, and embedded in Araldite (Fluka, Buchs, Switzerland). Ultrathin sections were prepared using a diamond knife, collected on copper grids ( G $300 \mathrm{Cu}$ ), contrasted using both lead citrate and uranyl acetate, and then examined with a "Zeiss EM 900" electron microscope.

2.3. Immunoelectron Microscopy. Control and AAPH-treated macrophages were fixed in $4 \%$ paraformaldehyde $+1 \%$ glutaraldehyde solution in $0.1 \mathrm{M}$ phosphate buffer ( $\mathrm{pH}$. 7.4) overnight at $4^{\circ} \mathrm{C}$. Cells were dehydrated at room temperature through graded ethanol solutions, then infiltrated in an intermediate solution of LR white acrylic resin (London Resin, Berkshire, UK) and 70\% ethanol $(2: 1)$ for $1 \mathrm{~h}$, and embedded in fresh LR white resin overnight at $4^{\circ} \mathrm{C}$. The resin was polymerized in gelatin capsules for $24 \mathrm{~h}$ at $50^{\circ} \mathrm{C}$. Ultrathin sections were prepared using a diamond knife and collected on Formvar carbon-coated nickel grids. For indirect immunolabeling, grids with sections were floated on drops of $1 \%$ bovine serum albumin in PBS containing $0.02 \mathrm{M}$ glycine and normal goat serum at room temperature for $30 \mathrm{~min}$ to reduce nonspecific binding. Sections were successively incubated with a rabbit polyclonal antibody to beclin-1 (1:10) (Santa Cruz Biotechnology, Heidelberg, Germany) in PBS $+0.1 \%$ BSA at $4^{\circ} \mathrm{C}$ overnight. After the grids had been washed rigorously several times with drops of PBS $+0.1 \%$ BSA, they were then incubated with 10 $\mathrm{nm} \gamma$-globulin goat antirabbit-gold particle complex at a dilution of $1: 10$ for $1 \mathrm{~h}$ at room temperature. Control staining to demonstrate immunohistochemical specificity included replacement of primary antibody by nonimmune goat serum at $4^{\circ} \mathrm{C}$ overnight. After immunolabeling, the sections were washed with PBS + 0.1\% BSA, further washed with distilled water, dried, and then stained with uranyl acetate. All sections were examined with a "Zeiss EM 900" electron microscope.

\section{Results}

3.1. Ultrastructural Analysis of Monocyte-Derived Human Macrophages Following Oxidative Stress. The ultrastructure of cultured blood monocyte-derived human macrophages was investigated and correlated under the effect of AAPHinduced oxidative stress $($ dose $=5 \mathrm{mmol} / \mathrm{L})$.

Control macrophages were irregular in shape with a finely vacuolated cytoplasm and numerous folds, processes and pseudopodia pushed out in all directions. Cells showed prominent profiles of rough endoplasmic reticulum and Golgi apparatus. Mitochondria were characteristically abundant and easily distinguishable. They were either elongated or rounded with simply tubular cristae. Vacuolar and granular structures, probably lysosomal in nature, were only poor developed. The nucleus occupied an eccentric position within the cell and appeared rounded or ovoid in shape with a thin rim of condensed chromatin (Figures 1(a) and 1(b)).

In AAPH-treated groups, the cell size remained almost unchanged, nuclei displayed a smooth surface and a normal ellipsoidal shape with well-preserved nucleoplasmic and nucleolar components. The nuclear envelope also remained intact (Figure 2(a)).

The most striking feature evoked by oxidative stress was the increase in the number of lysosomes accompanied by 


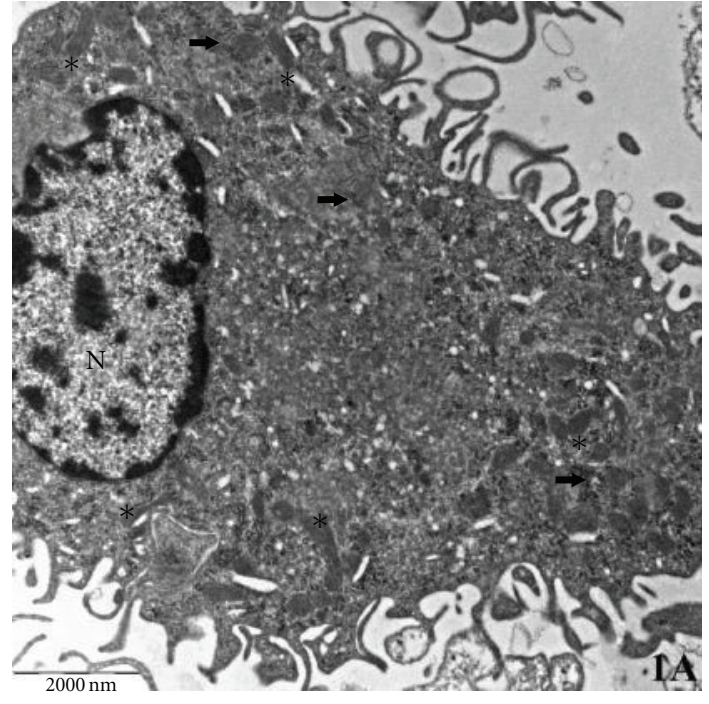

(a)

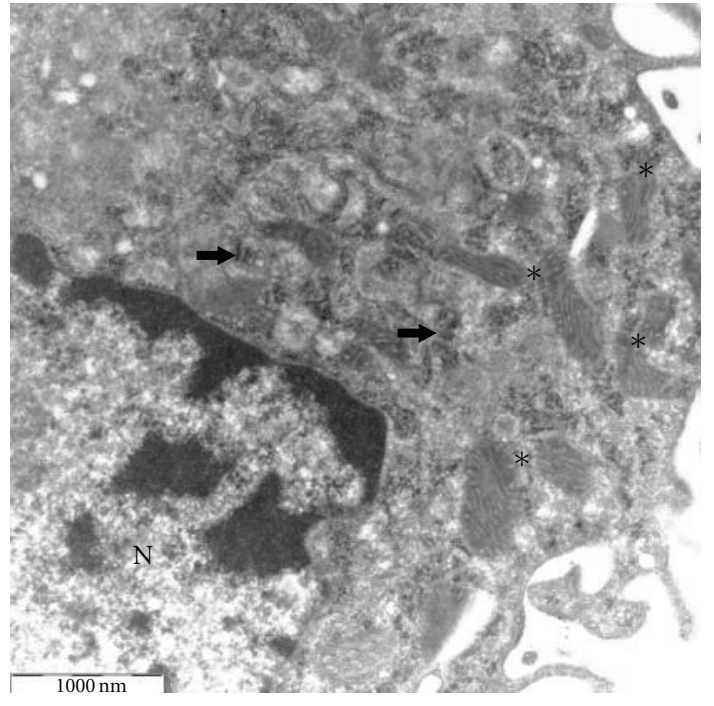

(b)

FIGURE 1: Ultrastructure of a normal, untreated human monocyte-derived macrophage. The cell possesses a finely vacuolated cytoplasm and, usually, numerous pseudopodia. The cytoplasm contains large mitochondria (asterisks) and a variable amount of free ribosomes and rough endoplasmic reticulum $\mathrm{rER}$ (arrows). $((\mathrm{a}) \times 10000$; (b) $\times 25000)$. N: nucleus.

the presence of multiple, cytoplasmic membrane-bound vesicles containing portions of the cytosol and membrane-like structures, probably from sequestered cytoplasmic organelles (Figures 2(b) and 2(c)). These structures were diagnostic of autophagy. In some cases, the sequestered material appeared ultrastructurally intact, while in other cases it showed loss of distinct morphological features as a sign of ongoing degradation. The alterations described above tended to vary in degree from cell to cell. In some macrophages, the cytoplasm was almost filled with autophagic vacuoles, containing electrondense elements and partially degraded material; intracellular organelles were also greatly reduced in number (Figure 3(a)). In contrast, other macrophages showed only moderate amounts of autophagic vacuoles, and cell organelles tended to accumulate in normal cytoplasmic areas (Figure 3(b)). Treated cells did not show morphological evidence suggestive of apoptosis, such as nuclear shrinkage, margination and condensation of the chromatin, enlargement of endoplasmic reticulum cisternae, and convolution of the cell with formation of apoptotic bodies.

\subsection{Analysis of Oxidative Stress-Induced Autophagy Using} Immunogold EM. Antibody against beclin-1 was used to precisely define the subcellular localization of the protein of interest in both basal conditions and upon oxidative stress. Rabbit pAb to human beclin-1 recognized only the $60 \mathrm{KDa}$ beclin-1 protein of mouse, rat and human origin, and no additional proteins. A distinct cytoplasmic labeling was found in normal, untreated macrophages (Figures 4(a) and 4(b)). Mitochondria and RER were the only significantly labelled elements in the cytoplasm. Beclin-1 immunolabeling was noted on the RER membranes and in the spaces between them. In the mitochondria, gold particles were associated with the outer, as well as the inner, mitochondrial membrane.
The cell nucleus was not labelled. To explore whether beclin1 was involved in the process of cell autophagy under conditions of oxidative stress, cultured macrophages were exposed to $5 \mathrm{mmol} / \mathrm{L}$ AAPH. Immunogold analysis revealed translocalization of beclin-1 to the autophagic vesicles (Figures 5(a) and 5(b)). The protein was mainly localized to the membranes of autophagic bodies; however, some gold particles were also found inside the lumen of these structures. The staining was seen in autophagic vesicles containing mitochondria, membrane-like structures, or other degenerated material. Almost no gold markers were present on RER cisternae and in the nucleus. Also the mitochondria were totally free of labelling. Beclin-1 immunoreaction was specific because (i) there was no labeling in the absence of the primary antibody and (ii) the reaction was strictly confined to the cells and only to specific compartments (iii) with no signal on the embedding medium.

\section{Discussion}

Oxidative stress, which is mediated by reactive oxygen and nitrogen species (ROS and RNS, resp.) has been increasingly related to the onset and/or progression of many human disorders ranging from cardiovascular to neurodegenerative and inflammatory diseases [20]. ROS and RNS are known to interact with cellular biomolecules such as membrane lipids, proteins, and nucleic acids, thereby resulting in alteration and/or disruption of cellular functions and membrane integrity and eventually leading to cell death [21, 22]. Accumulation of reactive species has also been proposed to induce autophagy $[23,24]$. To date, however, the molecular machinery that promotes autophagy during oxidative stress is still very poorly understood. 


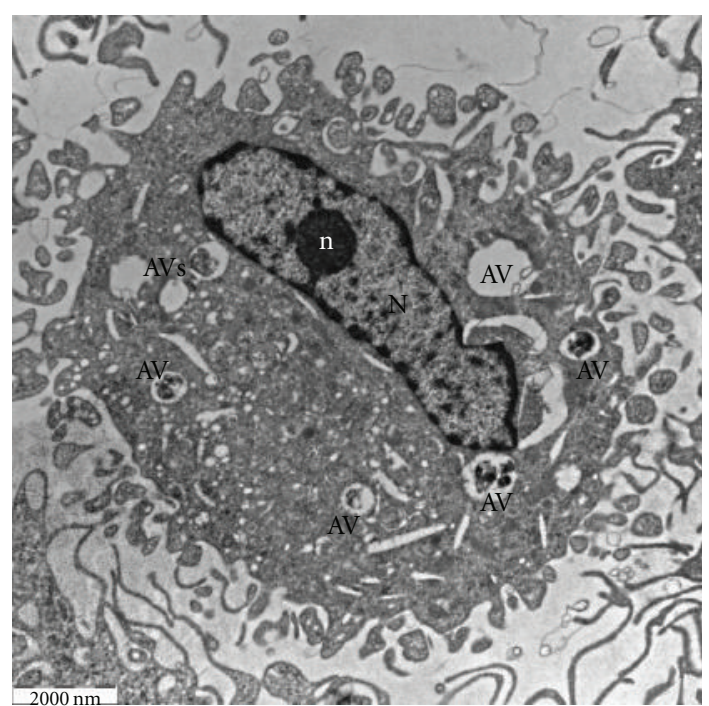

(a)

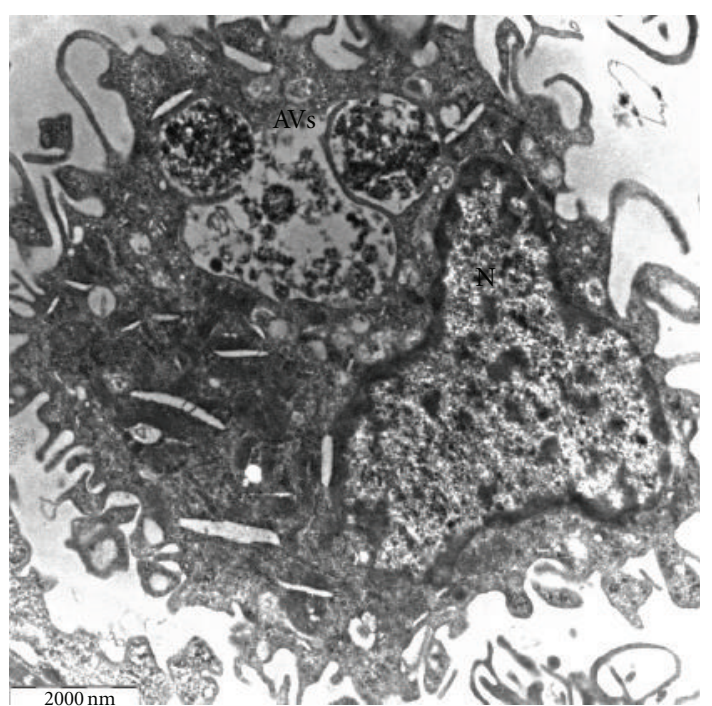

(b)

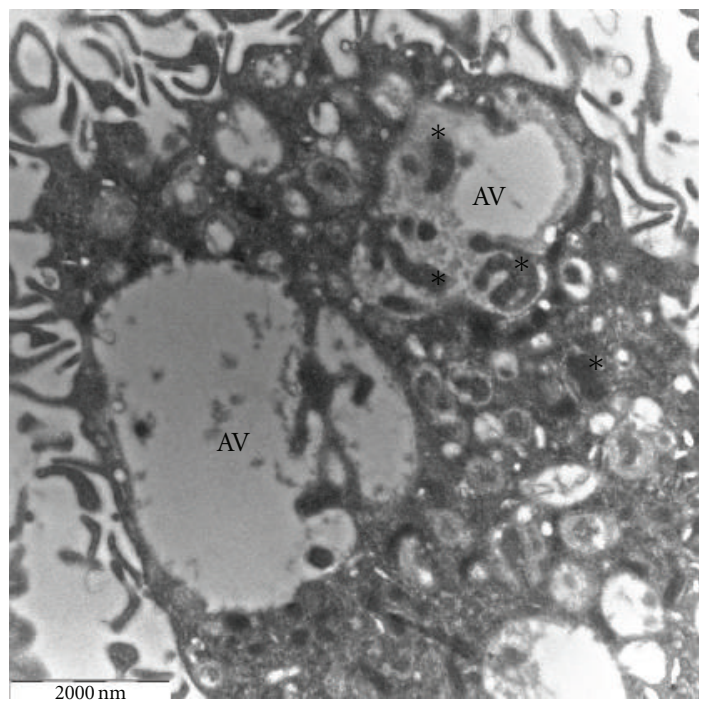

(c)

FIGURE 2: Ultrastructure of AAPH-treated macrophages. Nuclei appear intact with well-preserved nucleoli, a distinct nuclear envelope, and normal chromatin structure $((\mathrm{a}) \times 8000)$. Cells show the presence of many autophagic vacuoles of different sizes that enclose cytoplasmic components $((\mathrm{b}) \times 10000 ;(\mathrm{c}) \times 12500)$. N: nucleus; n: nucleolus; AV: autophagic vesicle; asterisks indicate mitochondria.

Herein, we demonstrate, that human monocyte-derived macrophages (HMDMs) exposed to AAPH undergo an active autophagic activity characterized by the formation of numerous membrane-bounded vesicles analogous to the autophagosomes previously described in various mammalian cells, plants, and yeast system [25-28]. Furthermore, we show that under conditions of oxidative stress induction of autophagy correlates with a redistribution of Beclin-1, an autophagy-related protein, from the cytoplasm to the autophagosomes.

AAPH is a well-known trigger of free radical-mediated oxidative reactions capable of inducing hemolysis, apoptosis, cell death, and enzyme inactivation because of its unique ability to generate peroxyl radicals. Besides the above mentioned effects, addition of AAPH to a cell culture system has been reported to increase intracellular ROS concentration and lipid hydroperoxide levels in cellular membrane resulting in oxidative stress [29].

Cellular oxidative stress has been shown to serve as an important stimulus for autophagy during periods of nutrient starvation, ischemia/reperfusion, hypoxia, and in response to cell stress promoting either cell survival and/or death $[18,30,31]$. Under conditions of oxidative stress, ROS are generated at high enough levels to cause oxidation and damage to DNA, lipids, proteins, and other cell constituents. Previous reports have proposed that increased autophagy may represent a second level of defense, when antioxidant activities are compromised, providing a selective advantage to minimize the effects of oxidative stress and conferring resistance to oxidant insults [32]. In this context, autophagy 


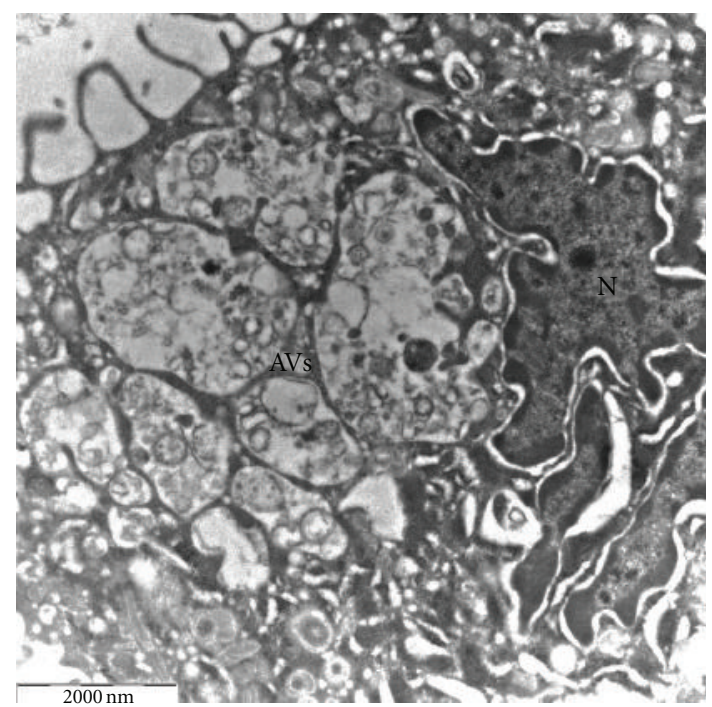

(a)

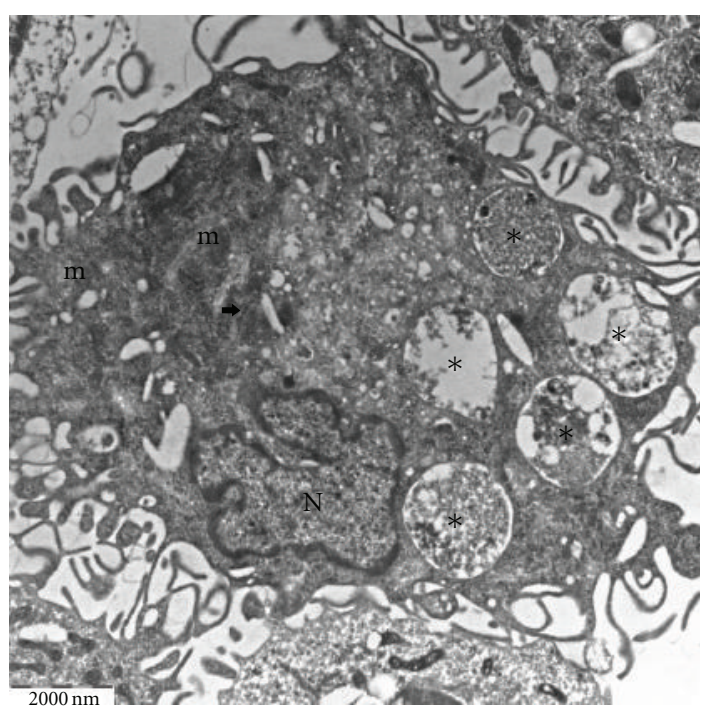

(b)

FIGURE 3: In the AAPH-treated groups, some cells appear to consist almost entirely of autophagic vesicles (asterisks), while others contain only a few vacuoles $((\mathrm{a}) \times 12500$; (b) $\times 8000)$. N: nucleus; m: mitochondria; arrow indicates RER.

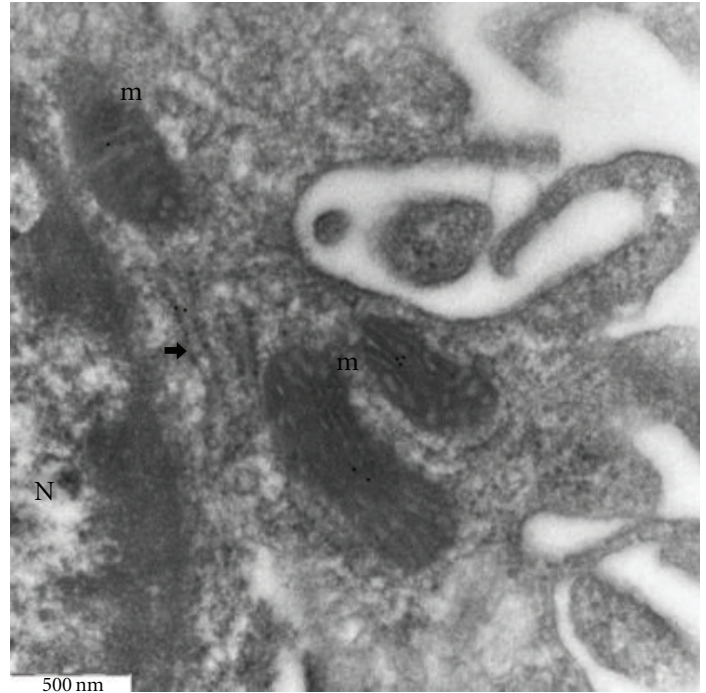

(a)

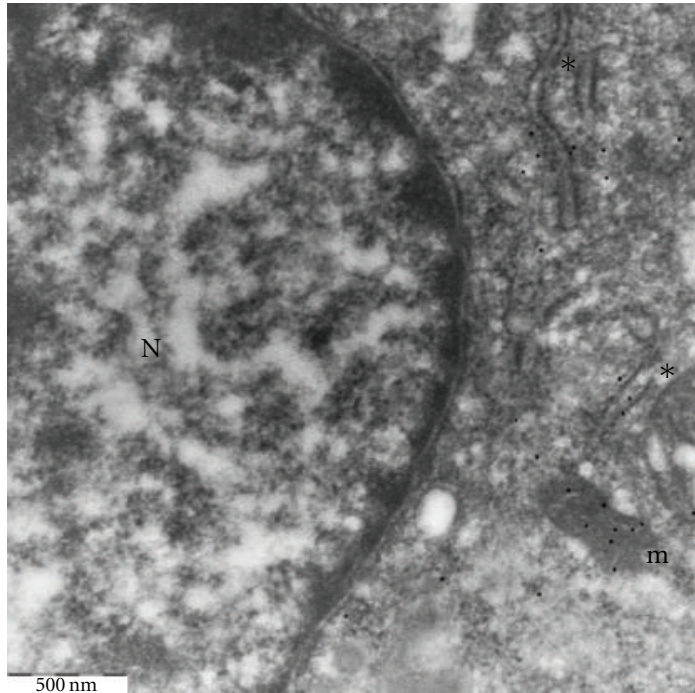

(b)

FIGURE 4: Immunoelectron microscopy of beclin-1 in control macrophages. Gold particles are present within the cytoplasm and organelles, such as rER (asterisks) and mitochondria but not in the nucleus $((\mathrm{a}) \times 50000 ;(\mathrm{b}) \times 40000)$. N: nucleus; m: mitochondria; arrow indicates RER.

may significantly promote cell survival by removing damaged cytosolic components, such as ribosomes (ribophagy), peroxisomes (micropexophagy and macropexophagy), endoplasmic reticulum (reticulophagy), parts of the nucleus (piecemeal microautophagy of the nucleus), and even mitochondria (mitophagy) [33].

In addition to a cell-autonomous role for autophagy in promoting survival, accumulating data suggest that autophagy may also regulate programmed cell death $[34,35]$. For instance, it has been demonstrated that after LPS treatment, and in the presence of caspase inhibitors, ROS can induce membrane blebbing and autophagic cell death in cultured macrophages through a caspase-independent pathway [36]. It has been also shown that under oxidative stress blockage of caspase, activation may trigger an "autophagy-related vicious circle" which enhances intracellular ROS levels via the selective degradation of catalase leading to a further increase in ROS and finally to autophagic cell death in mouse fibroblasts [37]. More recently, other studies showed that increased oxidative stress results in the induction of endoplasmic 


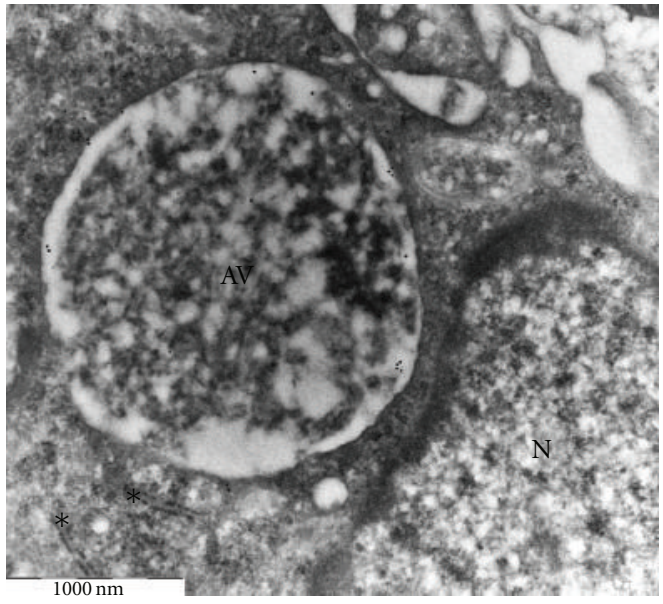

(a)

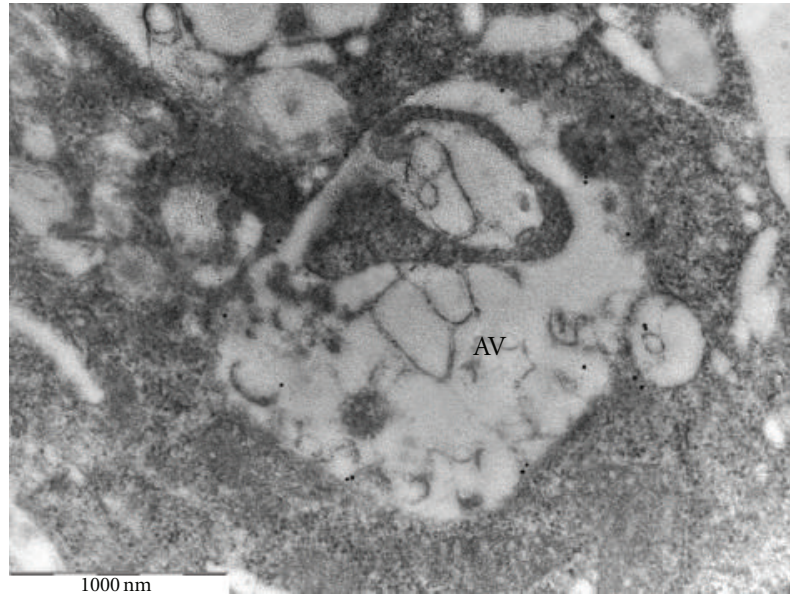

(b)

FIGURE 5: Immunoelectron microscopy of beclin-1 in AAPH-treated macrophages. Gold particles specifically localize to autophagic vesicles. Label occurs predominantly over the enveloping membranes $((a) \times 31500 ;(b) \times 31500)$. N: nucleus; AV: autophagic vesicle; asterisks indicate RER.

reticulum stress, which, in turn, can lead to autophagic cell death through the activation of the JNK/p38 signaling pathway [38].

Although different approaches have been applied to detect autophagy, electron microscopy still remains the "gold standard" method for assessing this phenomenon [39]. Unlike apoptosis, autophagy-mediated cell death pathway occurs whithout caspases activation, and therefore the nucleus appears intact and cellular fragmentation is absent [40]. On the basis of these criteria, we demonstrate by ultrastructural analysis, that under conditions of oxidative stress macrophages enlist autophagy accumulate autophagic vesicles but do not show morphologic hallmarks of apoptosis. Apart from being involved in the innate and adaptative immune response to bacteria, viruses, and parasites [41, 42], macrophages autophagy has been implicated in the pathogenesis of various oxidative stress-related diseases such as Crohn's disease (CD) and atherosclerosis. For instance, it has been demonstrated that defective autophagy modifies the handling of intracellular bacteria in Crohn's granulomas, while in the atherosclerotic plaque autophagy it may play role in the selective clearance of macrophages, thus, representing a potential mechanism of lesion stabilization [43-45].

In conclusion, the data presented here provide the first ultrastructural documentation that oxidative stress alters the subcellular distribution of beclin-1 and suggest that the protein may be actively involved in the regulation of autophagy by participating in the mechanism of autophagosomes formation. We also demonstrate that autophagy is characteristic of the response of macrophages to oxidative insults and presumably, in this scenario, it may promote the selective clearance of dysfunctional proteins and organelles. The threshold between autophagy as prosurvival or prodeath process is difficult to establish and probably depends on the rate and extent of degradation of cellular components upon oxidant exposure. Since excess or prolonged autophagy has been proved to lead to autophagic cell death, the dramatic increase of autophagic vacuoles frequently observed in our series might represent an overstimulation of this process, an irreversible step indicative of a final cell demise. From our and similar studies, the current consensus is that autophagy possesses both adaptive and maladaptive features, depending on the degree of activation and nature and duration of the injury with the final conclusion that additional studies are necessary to better understand many mechanistic aspects of this pathway and its dual role in life and death.

\section{References}

[1] C. de Duve and R. Wattiaux, "Functions of lysosomes," Annual Review of Physiology, vol. 28, pp. 435-492, 1966.

[2] N. Zhang, Y. Chen, R. Jiang et al., "PARP and RIP 1 are required for autophagy induced by 11'-deoxyverticillin A, which precedes caspase-dependent apoptosis," Autophagy, vol. 7, no. 6, pp. 598-612, 2011.

[3] N. Mizushima, "Autophagy: process and function," Genes and Development, vol. 21, no. 22, pp. 2861-2873, 2007.

[4] D. J. Klionsky and S. D. Emr, "Autophagy as a regulated pathway of cellular degradation," Science, vol. 290, no. 5497, pp. 1717-1721, 2000.

[5] C. Liang, "Negative regulation of autophagy," Cell Death and Differentiation, vol. 17, no. 12, pp. 1807-1815, 2010.

[6] Y. Chen and D. Klionsky, "The regulation of autophagyunanswered questions," Journal of Cell Science, vol. 124, no. 2, pp. 161-170, 2011.

[7] B. Ravikumar, S. Sarkar, J. E. Davies et al., "Regulation of mammalian autophagy in physiology and pathophysiology," Physiological Reviews, vol. 90, no. 4, pp. 1383-1435, 2010.

[8] C. W. Wang and D. J. Klionsky, "The molecular mechanism of autophagy," Molecular Medicine, vol. 9, no. 3-4, pp. 65-76, 2003.

[9] S. F. Funderburk, Q. J. Wang, and Z. Yue, "The Beclin 1-VPS34 complex-at the crossroads of autophagy and beyond," Trends in Cell Biology, vol. 20, no. 6, pp. 355-362, 2010.

[10] J. Q. Zhang, Y. M. Li, T. Liu et al., "Antitumor effect of matrine in human hepatoma G2 cells by inducing apoptosis and 
autophagy," World Journal of Gastroenterology, vol. 16, no. 34, pp. 4281-4290, 2010.

[11] X. H. Liang, J. Yu, K. Brown, and B. Levine, "Beclin 1 contains a leucine-rich nuclear export signal that is required for its autophagy and tumor suppressor function," Cancer Research, vol. 61, no. 8, pp. 3443-3449, 2001.

[12] Q. Sun, W. Fan, K. Chen, X. Ding, S. Chen, and Q. Zhong, "Identification of Barkor as a mammalian autophagy-specific factor for Beclin 1 and class III phosphatidylinositol 3-kinase," Proceedings of the National Academy of Sciences of the United States of America, vol. 105, no. 49, pp. 19211-19216, 2008.

[13] S. Hussey, M. R. Terebiznik, and N. L. Jones, "Autophagy: healthy eating and self-digestion for gastroenterologists," Journal of Pediatric Gastroenterology and Nutrition, vol. 46, no. 5, pp. 496-506, 2008.

[14] M. Djavaheri-Mergny, M. Amelotti, J. Mathieu, F. Besançon, C. Bauvy, and P. Codogno, "Regulation of autophagy by NF $\kappa \mathrm{B}$ transcription factor and reactives oxygen species," Autophagy, vol. 3, no. 4, pp. 390-392, 2007.

[15] S. Sakon, X. Xue, M. Takekawa et al., "NF- $\kappa$ B inhibits TNFinduced accumulation of ROS that mediate prolonged MAPK activation and necrotic cell death," EMBO Journal, vol. 22, no. 15, pp. 3898-3909, 2003.

[16] K. Nose, "Role of reactive oxygen species in the regulation of physiological functions," Biological and Pharmaceutical Bulletin, vol. 23, no. 8, pp. 897-903, 2000.

[17] M. Mancuso, D. Orsucci, A. Logerfo et al., "Oxidative stress biomarkers in mitochondrial myopathies, basally and after cysteine donor supplementation," Journal of Neurology, vol. 257, no. 5, pp. 774-781, 2010.

[18] M. B. Azad, Y. Chen, and S. B. Gibson, "Regulation of autophagy by reactive oxygen species (ROS): implications for cancer progression and treatment," Antioxidants and Redox Signaling, vol. 11, no. 4, pp. 777-790, 2009.

[19] M. Lopez, C. Martinache, S. Canepa, M. Chokri, F. Scotto, and J. Bartholeyns, "Autologous lymphocytes prevent the death of monocytes in culture and promote, as do GM-CSF, IL-3 and M-CSF, their differentiation into macrophages," Journal of Immunological Methods, vol. 26, no. 159, pp. 29-38, 1993.

[20] A. M. de la Villehuchet, M. Brack, G. Dreyfus et al., "A machine-learning approach to the prediction of oxidative stress in chronic inflammatory disease," Redox Report, vol. 14, no. 1, pp. 23-33, 2009.

[21] A. T. Hoye, J. E. Davoren, P. Wipf, M. P. Fink, and V. E. Kagan, "Targeting mitochondria," Accounts of Chemical Research, vol. 41, no. 1, pp. 87-97, 2008.

[22] S. W. Ryter, H. P. Kim, A. Hoetzel et al., "Mechanisms of cell death in oxidative stress," Antioxidants and Redox Signaling, vol. 9, no. 1, pp. 49-89, 2007.

[23] H. Wang, J. Ma, Y. Tan et al., "Amyloid- $\beta 1-42 \mathrm{M}$ induces reactive oxygen species-mediated autophagic cell death in U87 and SH-SY5Y cells," Journal of Alzheimer's Disease, vol. 21, no. 2, pp. 597-610, 2010.

[24] H. Yuan, C. N. Perry, C. Huang et al., "LPS-induced autophagy is mediated by oxidative signaling in cardiomyocytes and is associated with cytoprotection," American Journal of Physiology-Heart and Circulatory Physiology, vol. 296, no. 2, pp. H470-H479, 2009.

[25] S. Aubert, E. Gout, R. Bligny et al., "Ultrastructural and biochemical characterization of autophagy in higher plant cells subjected to carbon deprivation: control by the supply of mitochondria with respiratory substrates," Journal of Cell Biology, vol. 133, no. 6, pp. 1251-1263, 1996.
[26] M. Baba, K. Takeshige, N. Baba, and Y. Ohsumi, "Ultrastructural analysis of the autophagic process in yeast: detection of autophagosomes and their characterization," Journal of Cell Biology, vol. 124, no. 6, pp. 903-913, 1994.

[27] W. A. Dunn Jr., "Studies on the mechanisms of autophagy: formation of the autophagic vacuole," Journal of Cell Biology, vol. 110, no. 6, pp. 1923-1933, 1990.

[28] F. Marty, "Mise en Evidence d'un appareil provacuolaire et de son rfle dans l'utophagie cenulaire et l'origine des vacuoles," Comptes Rendus de l'Académie des Sciences, vol. 276D, pp. 1549-1552, 1973.

[29] R. Piga, Y. Saito, Y. Yoshida, and E. Niki, "Cytotoxic effects of various stressors on PC12 cells: involvement of oxidative stress and effect of antioxidants," NeuroToxicology, vol. 28, no. 1, pp. 67-75, 2007.

[30] N. Gurusamy and D. K. Das, "Autophagy, redox signaling, and ventricular remodeling," Antioxidants and Redox Signaling, vol. 11, no. 8, pp. 1975-1988, 2009.

[31] Y. Chen, E. McMillan-Ward, J. Kong, S. J. Israels, and S. B. Gibson, "Oxidative stress induces autophagic cell death independent of apoptosis in transformed and cancer cells," Cell Death and Differentiation, vol. 15, no. 1, pp. 171-182, 2008.

[32] M. N. Moore, "Autophagy as a second level protective process in conferring resistance to environmentally-induced oxidative stress," Autophagy, vol. 16, no. 2, pp. 254-256, 2008.

[33] W. L. Yen and D. J. Klionsky, "How to live long and prosper: autophagy, mitochondria, and aging," Physiology, vol. 23, no. 5, pp. 248-262, 2008.

[34] B. Levine and J. Yuan, "Autophagy in cell death: an innocent convict?" Journal of Clinical Investigation, vol. 115, no. 10, pp. 2679-2688, 2005.

[35] D. Gozuacik and A. Kimchi, "Autophagy as a cell death and tumor suppressor mechanism," Oncogene, vol. 23, no. 16, pp. 2891-2906, 2004.

[36] Y. Xu, S. O. Kim, Y. Li, and J. Han, "Autophagy contributes to caspase-independent macrophage cell death," Journal of Biological Chemistry, vol. 281, no. 28, pp. 19179-19187, 2006.

[37] L. Yu, F. Wan, S. Dutta et al., "Autophagic programmed cell death by selective catalase degradation," Proceedings of the National Academy of Sciences of the United States of America, vol. 103, no. 13, pp. 4952-4957, 2006.

[38] T. Verfaillie, M. Salazar, G. Velasco, and P. Agostinis, "Linking ER stress to autophagy: potential implications for cancer therapy," International Journal of Cell Biology, vol. 2010, Article ID 930509, 19 pages, 2010.

[39] P. Ylä-Anttila, H. Vihinen, E. Jokitalo, and E. L. Eskelinen, "Monitoring autophagy by electron microscopy in Mammalian cells," Methods in Enzymology, vol. 452, pp. 143-164, 2009.

[40] A. L. Edinger and C. B. Thompson, "Death by design: apoptosis, necrosis and autophagy," Current Opinion in Cell Biology, vol. 16, no. 6, pp. 663-669, 2004.

[41] M. A. Delgado, R. A. Elmaoued, A. S. Davis, G. Kyei, and V. Deretic, "Toll-like receptors control autophagy," EMBO Journal, vol. 27, no. 7, pp. 1110-1121, 2008.

[42] M. G. Gutierrez, S. S. Master, S. B. Singh, G. A. Taylor, M. I. Colombo, and V. Deretic, "Autophagy is a defense mechanism inhibiting BCG and Mycobacterium tuberculosis survival in infected macrophages," Cell, vol. 119, no. 6, pp. 753-766, 2004.

[43] T. Elliott, N. Prescott, B. Hudspith et al., "Autophagy gene polymorphisms influence the interaction of $\mathrm{E}$ coli and macrophages in Crohn's disease," Gut, vol. 60, p. A61, 2011. 
[44] R. Caprilli and G. Frieri, "The dyspeptic macrophage 30 years later: an update in the pathogenesis of Crohn's disease," Digestive and Liver Disease, vol. 41, no. 2, pp. 166-168, 2009.

[45] S. Verheye, W. Martinet, M. M. Kockx et al., "Selective clearance of macrophages in atherosclerotic plaques by autophagy," Journal of the American College of Cardiology, vol. 49, no. 6, pp. 706-715, 2007. 


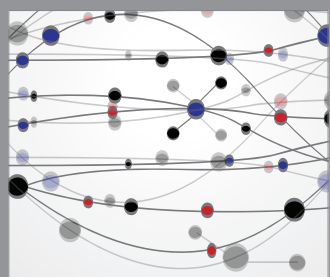

The Scientific World Journal
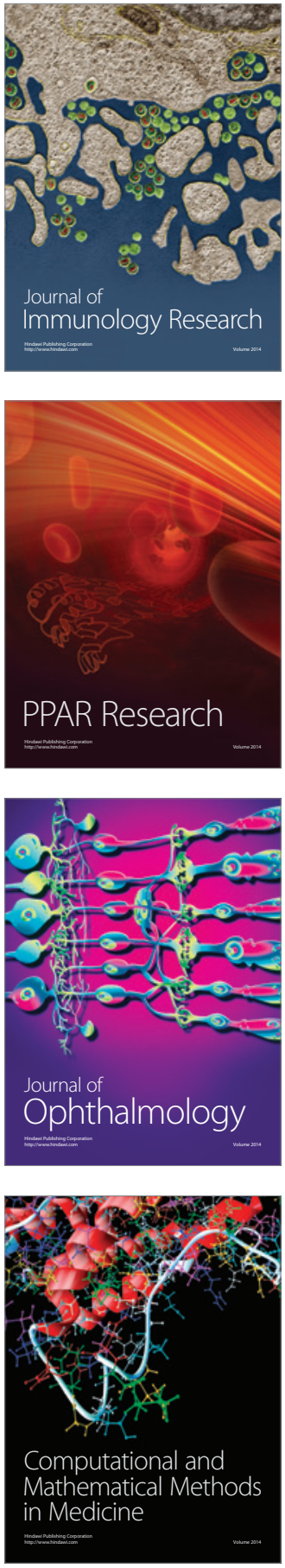

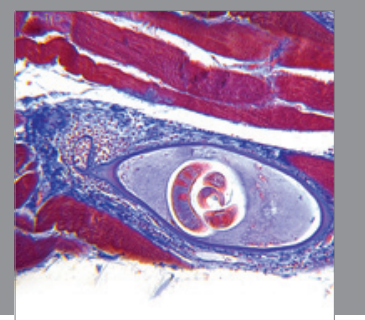

Gastroenterology

Research and Practice
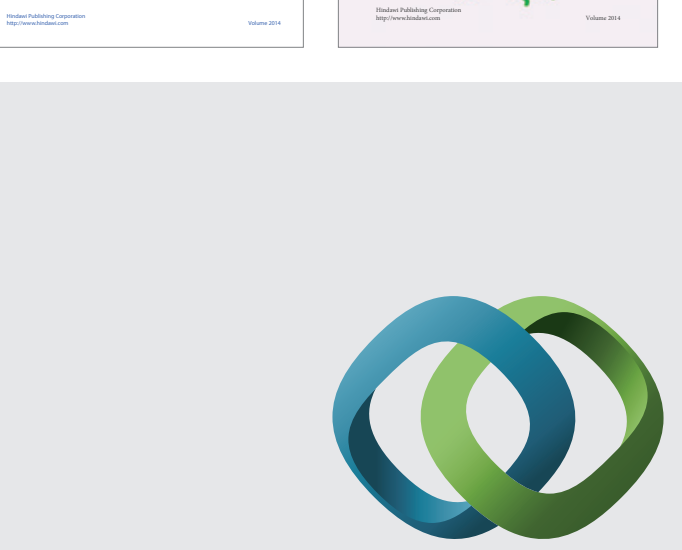

\section{Hindawi}

Submit your manuscripts at

http://www.hindawi.com
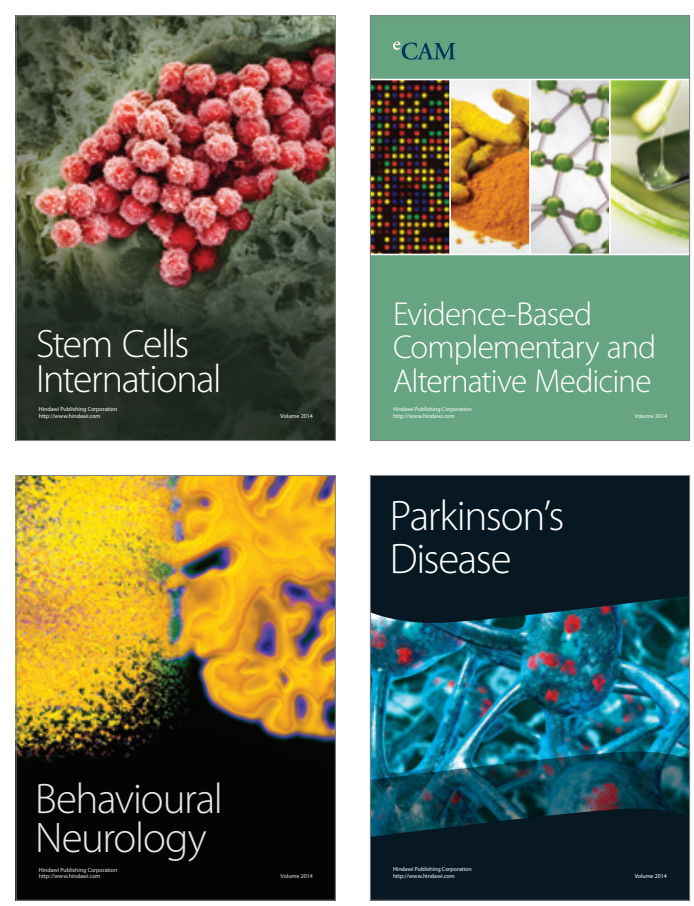

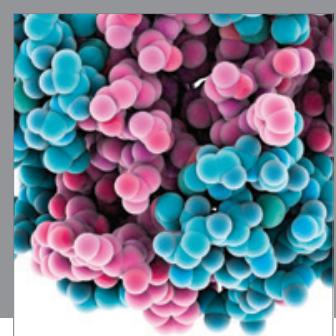

Journal of
Diabetes Research

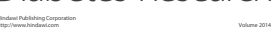

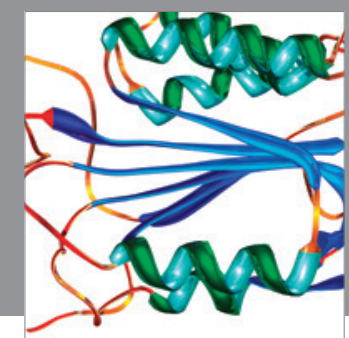

Disease Markers
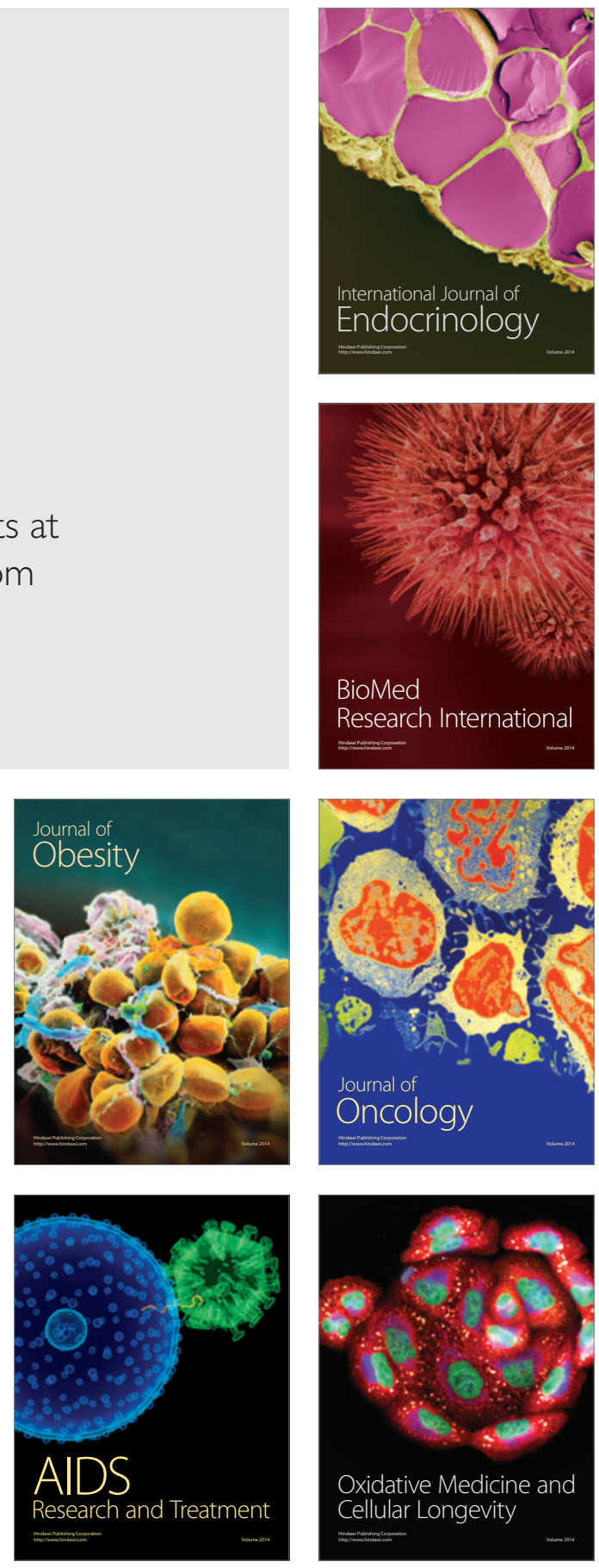